\title{
Experimental evidence of independence of nuclear de-channeling length on the particle charge sign
}

\author{
E. Bagli ${ }^{1, \text { a }}$, V. Guidi ${ }^{1}$, A. Mazzolari ${ }^{1}$, L. Bandiera ${ }^{1}$, G. Germogli ${ }^{1}$, A. I. Sytov ${ }^{1}$, D. De Salvador ${ }^{2,3}$, A. Berra ${ }^{4,5}$, \\ M. Prest ${ }^{4,5}$, E. Vallazza ${ }^{6}$ \\ ${ }^{1}$ INFN Sezione di Ferrara, Dipartimento di Fisica e Scienze della Terra, Università di Ferrara, Via Saragat 1, 44122 Ferrara, Italy \\ 2 Dipartimento di Fisica e Astronomia, Università di Padova, Via Marzolo 8, 35131 Padua, Italy \\ ${ }^{3}$ INFN Laboratori Nazionali di Legnaro, Viale dell'Università 2, 35020 Legnaro, Italy \\ ${ }^{4}$ Università dell'Insubria, via Valleggio 11, 22100 Como, Italy \\ ${ }^{5}$ INFN Sezione di Milano Bicocca, Piazza della Scienza 3, 20126 Milan, Italy \\ ${ }^{6}$ INFN Sezione di Trieste, Via Valerio 2, 34127 Trieste, Italy
}

Received: 29 June 2016 / Accepted: 23 January 2017 / Published online: 4 February 2017

(c) The Author(s) 2017. This article is published with open access at Springerlink.com

\begin{abstract}
Under coherent interactions, particles undergo correlated collisions with the crystal lattice and their motion result in confinement in the fields of atomic planes, i.e. particle channeling. Other than coherently interacting with the lattice, particles also suffer incoherent interactions with individual nuclei and may leave their bounded motion, i.e., they de-channel. The latter is the main limiting factor for applications of coherent interactions in crystal-assisted particle steering. We experimentally investigated the nature of dechanneling of $120 \mathrm{GeV} / \mathrm{c} e^{-}$and $e^{+}$in a bent silicon crystal at H4-SPS external line at CERN. We found that while channeling efficiency differs significantly for $e^{-}(2 \pm 2 \%)$ and $e^{+}(54 \pm 2 \%)$, their nuclear de-channeling length is comparable, $(0.6 \pm 0.1) \mathrm{mm}$ for $e^{-}$and $(0.7 \pm 0.3) \mathrm{mm}$ for $e^{+}$. The experimental proof of the equality of the nuclear dechanneling length for positrons and electrons is interpreted in terms of similar dynamics undergone by the channeled particles in the field of nuclei irrespective of their charge.
\end{abstract}

In the last decade, a significant boost to the research on particle-crystal interactions was provided by the fabrication of uniformly bent crystals [1] with thickness along the beam suitable for experiments at high-energy [2,3]. Measurements proved the capability of channeling for manipulation of positively [4] and negatively [5,6] charged particle beams from $\mathrm{MeV}$ [7] up to hundreds of $\mathrm{GeV}[8,9]$, and for the generation of intense electromagnetic radiation from sub-GeV $[10,11]$ to hundreds-GeV electron beams [12]. Moreover, channeling effects were exploited for steering [13], collimation [14] and extraction [15] of relativistic beams in circular accelerators, as well as splitting and focusing of extracted beams [16],

a e-mail: bagli@fe.infn.it leading to the installation of two bent crystals in the Large Hadron Collider (LHC) for collimation purposes [17]. The crystals installed in the LHC were successfully tested at 6.5 $\mathrm{TeV} / \mathrm{c}$ and proved to reduce the beam losses in the whole ring [18].

Particles under channeling undergo coherent interactions with the crystalline nuclei of planes or axes. Coherent interactions have been interpreted in terms of a continuous potential by Lindhard [19], i.e. time-reversible particle dynamics is governed by the conservative time-independent electric potential generated by the ordered atomic lattice. However, other than the interaction with a crystal as a whole, particles naturally suffer interactions with individual nuclei and electrons, which may abruptly vary the particle kinetic energy. The latter is called de-channeling and is the main limiting factor for applications of channeling.

Experiments with thin crystals allowed one to measure the de-channeling intensity for some positive and negative particles. In particular, the comparison of the results obtained for $150 \mathrm{GeV} / \mathrm{c}$ negative pions with the experimental data for the de-channeling on atomic nuclei of $400 \mathrm{GeV} / \mathrm{c}$ positive particles hinted that the intensity of the two phenomena may have the same magnitude.

In this paper we experimentally investigate the nature of the de-channeling process by the same bent $\mathrm{Si}$ crystal with a particle and its anti-particle $\left(e^{-}\right.$and $\left.e^{+}\right)$at the same 120 $\mathrm{GeV} / \mathrm{c}$ beam energy. The experiments were carried out at the H4-SPS line at CERN.

The motion of channeled particles is affected by incoherent scattering processes with electrons and nuclei that cause the non-conservation of the transverse energy. As a consequence, the transverse energy may exceed the potential 
well barrier causing the particle to leave the channeling state, i.e., they are de-channeled. Dependently on their transverse energy, particles can either enter or not the nuclear corridor, i.e. the volume of the crystal within which nuclei perform their thermal vibration. The fraction of particles that have sufficient transverse energy to enter the nuclear corridor is $f_{\mathrm{n}}$ at the crystal entry face $(z=0)$. The remaining fraction is $f_{e}=1-f_{\mathrm{n}}$.

Due to strong interaction with nuclei, $f_{\mathrm{n}}$ rapidly shrinks, e-folding at distance $l_{\mathrm{n}}$ from the surface, which is called the nuclear de-channeling length. In order to estimate the fraction $f_{\mathrm{n}}$, the atomic density can be approximated by a Gaussian distribution with standard deviation equal to the atomic thermal vibration amplitude $\left(u_{t}\right)$ which is $0.075 \AA$ for $\mathrm{Si}$ at $273 \mathrm{~K}$ [20]. By assuming that the region with intense multiple scattering extends over $2.5 u_{t}$ [21] - the so-called nuclear corridor - and bearing in mind that (110) interplanar distance is $d_{\mathrm{p}}=1.92 \AA, \sim 19.5 \%$ of particles of a perfectly parallel beam are subject to nuclear de-channeling.

The remaining $f_{e}$ fraction of particles does not initially interact with nuclei and thereby undergoes interaction with electrons only. The interaction strengths with electrons and nuclei are quite different, e.g., for a collimated $400 \mathrm{GeV} / \mathrm{c}$ proton beam interacting with $\mathrm{Si}(110)$ crystal, $l_{e}^{(+)} \sim 220 \mathrm{~mm}$ [22], while $l_{\mathrm{n}}^{(+)} \sim 1.5 \mathrm{~mm}$ [21], with $l_{e}^{(+)}$being the electronic de-channeling length. The electronic de-channeling length scales proportionally to the particle momentum [23] and was extensively measured for protons $[13,24,25]$.

In the literature $[4,21,26]$, the channeled-particle fraction $f_{\text {ch }}^{(+)}$at depth $z$ in the crystal holds

$f_{\mathrm{ch}}^{(+)}(z) \approx f_{\mathrm{n}} e^{-z / l_{\mathrm{n}}^{(+)}}+f_{e} e^{-z / l_{e}^{(+)}}$

where $f_{\mathrm{ch}}$ is the fraction of channeled particles and $l_{e}^{(+)}$is the electronic de-channeling length, i.e. the distances after which a $1 / e$ fraction of the initial particles are still under channeling.

For negatively charged particles, since the minimum of the potential well is located on the atomic planes, the mechanism of electronic de-channeling has a negligible contribution $\left(f_{e} \sim 0, f_{\mathrm{n}} \sim 1\right)$ because all the particles do interact with nuclei $[5,6,26]$. Therefore, the channeled-particle fraction $f_{c h,-}$ holds,

$f_{\mathrm{ch}}^{(-)}(z) \approx e^{-z / l_{\mathrm{n}}^{(-)}}$.

A bent crystal is capable of separating channeled, neverchanneled, and de-channeled fractions $[21,26]$. Indeed, the channeled fraction is deflected to the nominal crystal bending angle, the never-channeled fraction is only scattered, while the de-channeled fraction results in a deflection angle lower than the nominal crystal bending angle. Therefore, for the measurement of $l_{\mathrm{n}}^{(+)}$and $l_{\mathrm{n}}^{(-)}$, a slightly bent thin crystal is the optimal choice, since the three particle fractions can easily be discriminated. Channeling efficiency decreases as the crystal curvature $1 / R$ increases, and vanishes for $R<R_{\mathrm{c}}, R_{\mathrm{c}}$ being the critical radius for channeling $[27,28]$. The usage of a crystal with $R \gg R_{\mathrm{c}}$ does not significantly lower the channeling efficiency. In fact, particles de-channeled at a crystal depth $z$ are deflected by an angle $\theta_{z} \approx z / R$, thereby a measure of the rate of de-channeled particles as a function of the crystal depth can be inferred $[21,25]$. Moreover, a crystal with $l \ll l_{e}$ allows one to distinguish the nuclear de-channeling length $l_{\mathrm{n}}$ for positive particles, since the contribution of the second term in Eq. 1 is very small.

The quantities $l_{e}^{(+)}$and $R_{\mathrm{c}}$ scale as particle momentumvelocity $p \beta$ [20]. For $120 \mathrm{GeV} / \mathrm{c}$ particle momentum, $l_{e}^{(+)}$ is $\approx 45 \mathrm{~mm}$ and $R_{\mathrm{c}}$ is $\approx 0.21 \mathrm{~m}$. A Si strip that fulfills the requirements $l \ll l_{e}$ and $R \gg R_{\mathrm{c}}$ was manufactured starting from prime-quality wafers. The strip was shaped as a parallelepiped of size $1.99 \times 55.0 \times 2.01 \mathrm{~mm}^{3}$, with $l_{\text {cry }}=2.01$ $\mathrm{mm}$ being the length along the beam direction, and was bent using a custom-made mechanical device [15], resulting in an anticlastic bending of the (110) planes with $R=(11.5 \pm 0.5)$ $\mathrm{m}$. The strip bending radius [29] was determined by means of interferometric and diffractometric measurements through a VEECO NT-1100 white-light interferometer and a Panalytical X-Pert MRD-PRO diffractometer, respectively.

The crystal was exposed to a $120 \mathrm{GeV} / \mathrm{c} e^{-}$and $e^{+}$beams at the H4 CERN-SPS extracted line with $(66 \pm 2) \times(97 \pm$ 5) $\mu \mathrm{rad}^{2}$ divergence rms. The holder with the crystal was mounted on a two-axis goniometer with an angular resolution of $\sim 1 \mu \mathrm{rad}$. The particle incoming and outgoing angles from the crystal were detected by means of a tracking detectors system [30]. The standard deviation of $14.6 \mu \mathrm{rad}$ for the angular resolution of the system was verified through Geant4 Monte Carlo simulations [31,32]. An electromagnetic calorimeter was positioned after the telescope system, allowing for the selection of $e^{-}$and $e^{+}$and the rejection of muons and hadrons. The strip largest face orthogonal to the $\langle 110\rangle$ axis was oriented parallel to the beam direction.

Figure 1a shows the experimental distributions of the deflection angle under channeling for $e^{-}$and $e^{+}$. Since particles undergo channeling when the angle $\theta$ between their direction and the crystal planes is smaller than the critical angle for channeling, $\theta_{\mathrm{c}}\left(18.8 \mu \mathrm{rad}\right.$ for $\left.120 \mathrm{GeV} / \mathrm{c} e^{+}\right)$, only the particles with $\theta<\theta_{\mathrm{c}} / 2$ were analyzed.

The analysis of the distributions was carried out using the fitting procedure described in Ref. [33] for $e^{-}$. The probability distribution of the de-channeling particles $\left(\mathrm{d} P_{\mathrm{d}} / \mathrm{d} \theta(\theta)\right)$ is

$$
\begin{aligned}
& \frac{\mathrm{d} f_{\mathrm{d}}}{\mathrm{d} \theta}\left(\theta, \theta_{\mathrm{d}}\right) \\
& \quad=\frac{1-f_{\mathrm{u}}}{2 \theta_{\mathrm{d}}} e^{\frac{\sigma_{\mathrm{u}}^{2}}{\theta_{\mathrm{d}}^{2}}+\frac{\theta_{\mathrm{c}}}{\theta_{\mathrm{d}}}-\frac{\theta}{\theta_{\mathrm{d}}}}\left(\operatorname{erf}\left(\frac{\theta_{\mathrm{u}}-\Delta \theta}{\sqrt{2} \sigma_{\mathrm{u}}}\right)-\operatorname{erf}\left(\frac{\theta_{\mathrm{c}}-\Delta \theta}{\sqrt{2} \sigma_{\mathrm{u}}}\right)\right)
\end{aligned}
$$




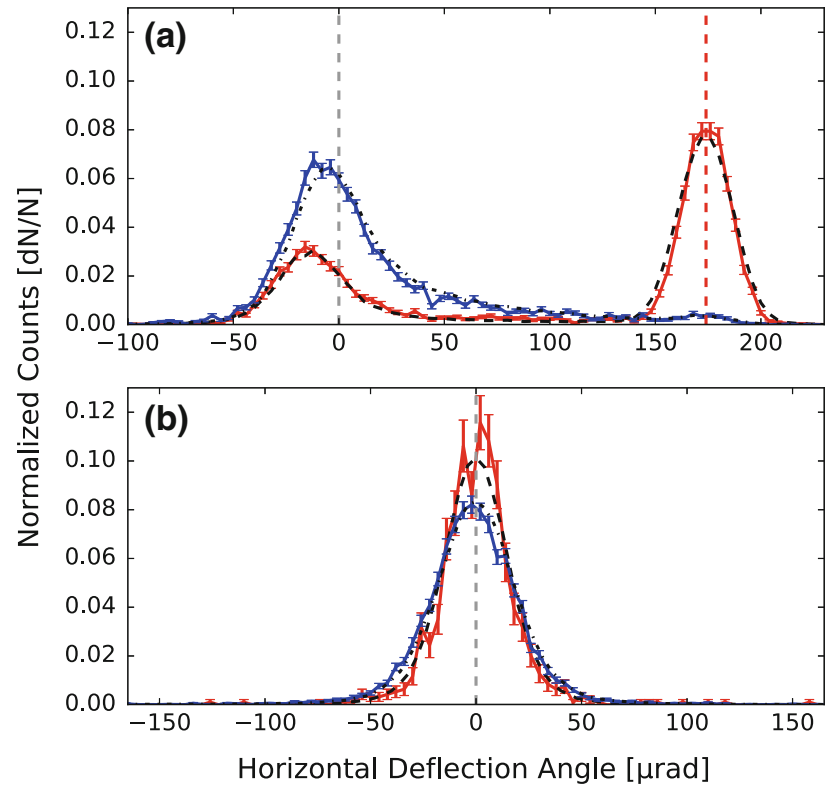

Fig. 1 Experimental measurements (red for $e^{+}$and blue for $e^{-}$) and Geant 4 simulations (black dashed line for $e^{+}$and black dash-dotted line for $e^{-}$) of the deflection-angle distribution in the bent (a) and free (b) directions for $e^{+}$and $e^{-}$beams interacting with the crystal. Only particles with an incoming angle less than half of the critical channeling angle, $\theta_{\mathrm{c}}\left(18.8 \mu \mathrm{rad}\right.$ for $\left.120 \mathrm{GeV} / \mathrm{c} e^{+}\right)$, with respect to the channeling plane have been analyzed

where $f_{\mathrm{u}}$ is the fraction of particles in the undeflected peak, $\sigma_{\mathrm{u}}$ and $\theta_{\mathrm{u}}$ the standard deviation and the mean of the distribution for the undeflected peak, $\theta_{\mathrm{c}}$ the mean of the distribution for the channeling peak, $\theta_{\mathrm{d}}=l_{\mathrm{n}} / l_{\mathrm{cry}} \theta_{\mathrm{c}}$ and $\Delta \theta=\theta-\sigma_{\mathrm{u}}^{2} / \theta_{\mathrm{d}}$. Figure $2 b$ shows the fitted distribution over the experimental one and the fit parameters are summarized in Table 1. The channeling efficiency is $(2 \pm 2) \%$ and the de-channeling length is $(0.6 \pm 0.1) \mathrm{mm}$.

The measured de-channeling length is shorter than the crystal length. As a consequence, since we suppose that the nuclear de-channeling lengths for positive and negative particles are similar, the fraction of particles de-channeled due to electronic de-channeling may be visible. Therefore, the probability distribution of the de-channeling tail for $e^{+}$becomes the sum of two terms:

$f_{e} \frac{\mathrm{d} f_{\mathrm{d}}}{\mathrm{d} \theta}\left(\theta, \theta_{e}\right)+f_{\mathrm{n}} \frac{\mathrm{d} f_{\mathrm{d}}}{\mathrm{d} \theta}\left(\theta, \theta_{\mathrm{n}}\right)$

where $\theta_{e}=l_{\mathrm{e}} / l_{\text {cry }} \theta_{\mathrm{c}}$ and $\theta_{\mathrm{n}}=l_{\mathrm{n}} / l_{\text {cry }} \theta_{\mathrm{c}}, f_{\mathrm{n}}$ and $f_{e}$ being the fraction of channeled particles under nuclear and electronic de-channeling, respectively. Figure 2a shows the fitted distribution over the experimental one and the fit parameters are summarized in Table 1 . The channeling efficiency is $(54 \pm 2) \%, l_{\mathrm{n}}^{(+)}=(0.7 \pm 0.3) \mathrm{mm}$ and $f_{\mathrm{n}}^{(+)}=(30 \pm 10) \%$. The electronic de-channeling length $l_{e}^{(+)}$was set to $44.6 \mathrm{~mm}$ [20]. The same fit procedure was repeated for the case of electrons, resulting in $f_{\mathrm{n}}^{(-)}=(100.0 \pm 0.1) \%$, which is consis-
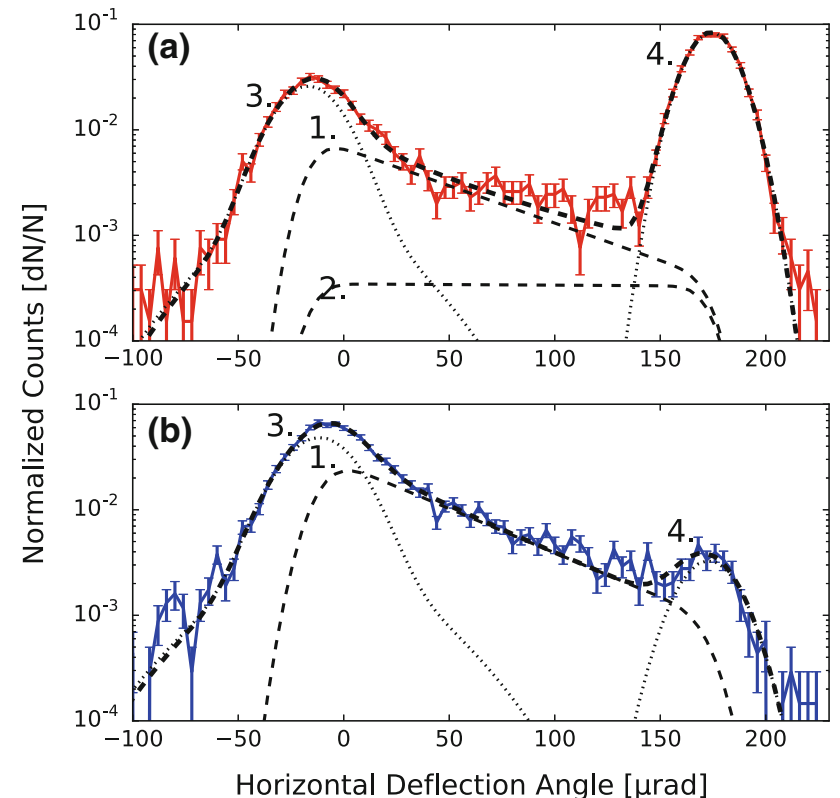

Fig. 2 Experimental measurements (a for $e^{+}$and $\mathbf{b} e^{-}$) and fitted distributions (black dash-dotted for the fitted distribution, dashed for the nuclear (1.) and electronic (2.) de-channeling components and dotted lines for the undeflected (3.) and channeling (4.)) of the deflection-angle distribution in the bent direction for $e^{+}$and $e^{-}$beams interacting with the crystal. Only particles with an incoming angle less than half of the critical channeling angle, $\theta_{\mathrm{c}}\left(18.8 \mu \mathrm{rad}\right.$ for $\left.120 \mathrm{GeV} / \mathrm{c} e^{+}\right)$, with respect to the channeling plane have been analyzed

tent with the initial supposition that negative particles are subject to nuclear de-channeling only. As previously noted, the rate of nuclear de-channeling depends on incoherent interactions with atomic nuclei, which are similar for positive and negative particles. In fact, measurements showed that $l_{\mathrm{n}}$ does not significantly vary with particle charge at all.

Figure 1b shows the experimental distributions of the deflection angle under channeling for $e^{-}$and $e^{+}$for the free direction, i.e. the direction not bent. The analysis of the distributions was carried out using the fitting procedure described in Ref. [33] and the fit parameters are summarized in Table 2. The scattering in the free plane for negative particles is stronger for the particles under channeling than for those not aligned with the crystal planes, while for positive particles the opposite occurs. Indeed for positive particles the largest fraction of the particles does not interact with the nuclei, reducing the probability of incoherent scattering.

Monte Carlo simulations were carried out using the Geant4 toolkit $[31,32]$. The experimental setup at the H4SPS area is reproduced in the simulation in order to take into account the error due to the finite resolution of the telescope. Channeling is implemented via an updated version of the Geant 4 channeling package [34]. The package does not take into account coherent radiation processes. The results are shown in Fig. 1 and the fit parameters are summarized 
Table 1 Fit parameters for the distribution of the deflection angles in the bent direction for $120 \mathrm{GeV} / \mathrm{c} e^{+}$and $e^{-}$after the interaction with the $2.01 \mathrm{~mm} \mathrm{Si}(110)$ crystal, where $f_{\mathrm{c}}, \theta_{\mathrm{c}}$ and $\sigma_{\mathrm{c}}$ and $f_{\mathrm{u}}, \theta_{\mathrm{u}}$ and $\sigma_{\mathrm{u}}$ are the efficiency, mean deflection and standard deviation of the channeling and undeflected peaks, $l_{\mathrm{n}}$ the nuclear de-channeling length and $f_{\mathrm{n}}$ the fraction of particles under nuclear de-channeling. The parameters $A$ and $r$ for the double Gaussian distribution of the undeflected peak were set equal to 0.89 and 2.3, as for the misaligned orientation (see Table 2), and $l_{e}$ was set to $44.6 \mathrm{~mm}[20]$

\begin{tabular}{llllllllll}
\hline Type & Particle & $f_{\mathrm{u}}(\%)$ & $\theta_{\mathrm{u}}(\mu \mathrm{rad})$ & $\sigma_{\mathrm{u}}(\mu \mathrm{rad})$ & $f_{\mathrm{c}}(\%)$ & $\theta_{\mathrm{c}}(\mu \mathrm{rad})$ & $\sigma_{\mathrm{c}}(\mu \mathrm{rad})$ & $l_{\mathrm{n}}(\mathrm{mm})$ & $f_{\mathrm{n}}(\%)$ \\
\hline Data & $e^{+}$ & $23 \pm 1$ & $-17 \pm 1$ & $8.2 \pm 0.1$ & $54 \pm 2$ & $174 \pm 2$ & $6.3 \pm 0.1$ & $0.7 \pm 0.3$ & $30 \pm 10$ \\
Geant4 & $e^{+}$ & $25 \pm 1$ & $-15 \pm 1$ & $8.6 \pm 0.1$ & $59 \pm 2$ & $174 \pm 2$ & $7.2 \pm 0.1$ & $0.7 \pm 0.2$ & $21 \pm 5$ \\
Data & $e^{-}$ & $41 \pm 2$ & $-11 \pm 1$ & $9.1 \pm 0.2$ & $2 \pm 2$ & $173 \pm 2$ & $7.5 \pm 0.7$ & $0.6 \pm 0.1$ & $100 \pm 0.1$ \\
Geant4 & $e^{-}$ & $33 \pm 2$ & $-10 \pm 1$ & $9.1 \pm 0.1$ & $2 \pm 2$ & $173 \pm 2$ & $8.5 \pm 0.3$ & $0.6 \pm 0.2$ & $100 \pm 0.1$ \\
\hline
\end{tabular}

Table 2 Fit parameters for the distribution of the deflection angles in the free direction after the interaction of $120 \mathrm{GeV} / \mathrm{c} e^{+}$and $e^{-}$with the $2.01 \mathrm{~mm} \mathrm{Si} \mathrm{(110)} \mathrm{crystal} \mathrm{under} \mathrm{channeling.} \mathrm{The} \mathrm{distribution} \mathrm{is} \mathrm{the} \mathrm{sum}$ of two Gaussians, where $A$ is the constant factor of the first Gaussian
( $1-A$ for the second Gaussian) and $r$ is the ratio between the standard deviations of the second Gaussian and the one of the first Gaussian. The same fit was carried out for particles not aligned with the crystal planes (not aligned) for both $e^{-}$and $e^{+}$

\begin{tabular}{lllrrr}
\hline Type & Condition & Particle $(\mu \mathrm{rad})$ & $\sigma$ & $A$ & \multicolumn{2}{c}{${ }^{2}$} \\
\hline Data & Channeling & $e^{+}$ & $7.6 \pm 0.4$ & $0.88 \pm 0.03$ & $2.7 \pm 0.1$ \\
Geant4 & Channeling & $e^{+}$ & $8.6 \pm 0.1$ & $0.91 \pm 0.02$ & $2.5 \pm 0.1$ \\
Data & Channeling & $e^{-}$ & $10.3 \pm 0.2$ & $0.87 \pm 0.02$ & $2.6 \pm 0.1$ \\
Geant4 & Channeling & $e^{-}$ & $10.2 \pm 0.1$ & $0.89 \pm 0.01$ & $2.3 \pm 0.1$ \\
Data & Not aligned & $e^{+} / e^{-}$ & $8.8 \pm 0.1$ & $0.89 \pm 0.02$ \\
\hline
\end{tabular}

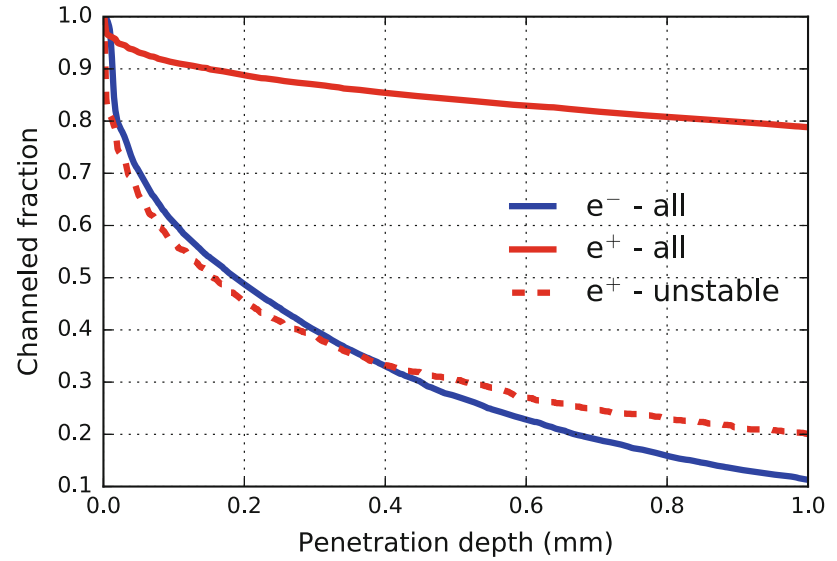

Fig. 3 Simulation of the fraction of particles under channeling interacting with a (110) Si straight crystal for $120 \mathrm{GeV} / \mathrm{c} e^{-}\left(e^{-}-\right.$all $), e^{+}$ $\left(e^{+}-\right.$all $)$and for the fraction of channeled $e^{+}$that impinge on the crystal close to the atomic planes $\left(e^{+}-\right.$unstable $)$

in Tables 1 and 2. Simulations show good agreement with experimental data for both positive and negative particles.

The availability of a Monte Carlo code for the simulations of the coherent phenomena allows one to have insight into the de-channeling mechanism. Indeed, other than comparing the deflection distribution at the exit of the crystal, the evolution of the beam phase space into the crystal can be studied. Figure 3 shows the evolution of the fraction of channeled particles as a function of the penetration depth into a straight crystal of $1 \mathrm{~mm}$. In the simulation $120 \mathrm{GeV} / \mathrm{c}$ $e^{-}$and $e^{+}$collimated beams impinge on a Si (110) crystal. The simulations were worked out via the DYNECHARM++ code $[35,36]$. As can be noticed, the fraction of particles in unstable channeling condition that impinge on the crystal close to the atomic planes, i.e. at a distance less than 2.5 times the amplitude of atomic thermal vibration, decreases as the fraction of channeled particles for a $e^{-}$beam. On the other hand, the channeling efficiency of the whole $e^{+}$beam remains higher than $80 \%$.

Figure 4 shows five snapshots of the evolution of the phase space for a perfectly collimated beam of $e^{+}$and $e^{-}$interacting with a $\mathrm{Si}$ (110) straight crystal for a lively representation of the particle dynamics (evolution sequence is available as supplementary material). The evolution of the $e^{+}$particles under stable channeling condition, i.e. that oscillate far from atomic plane, maintains a coherent pattern in the phase space for a period much longer than the $e^{-}$particles. For positive particles the length for which the confined channeled particles are randomly distributed in the phase-space spot is similar to $l_{\mathrm{n}}^{(+)}$, while the distribution of negative particles is immediately randomized approximately after a single oscillation period due to the strong interaction with atomic nuclei.

In summary, the nuclear de-channeling lengths of 120 $\mathrm{GeV} / \mathrm{c} e^{-}$and $e^{+}$were measured. A state-of-the-art slightly bent $\mathrm{Si}$ crystal was adopted to separate channeled, unchanneled and de-channeled fractions, resulting in the capability of measuring the rate of incoherent interactions with nuclei. We found that the channeling efficiency is different, $2 \pm 2 \%$ 


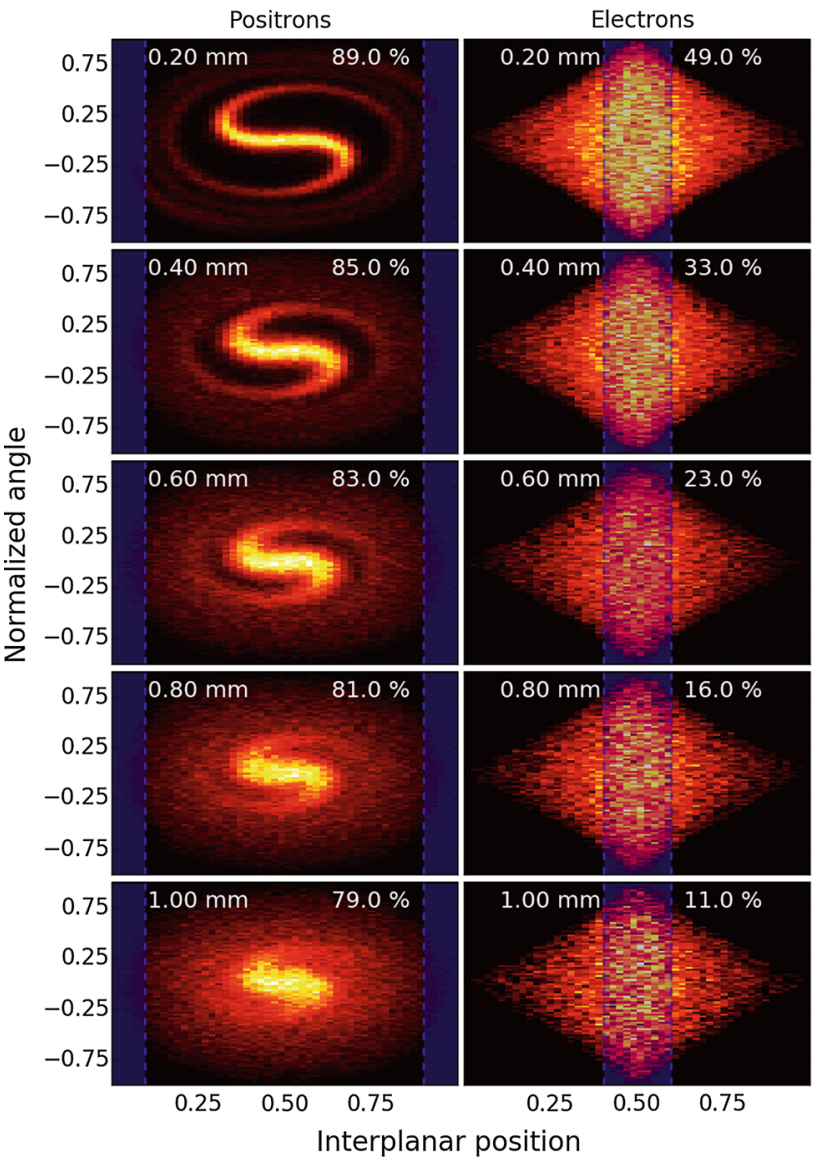

Fig. 4 Evolution of the phase space for a collimated beam of $e^{+}$and $e^{-}$ interacting with a $\mathrm{Si}(110)$ straight crystal. The full sequence is available as supplementary material. Top right and top left of each figure show the penetration depth at which the snapshots was recorded and the fraction of particles under channeling, i.e. the channeling efficiency

for $e^{-}$and $54 \pm 2 \%$ for $e^{+}$, while the nuclear de-channeling length is comparable, $l_{\mathrm{n}}^{(-)}$and $l_{\mathrm{n}}^{(+)}$being $(0.6 \pm 0.1) \mathrm{mm}$ and $(0.7 \pm 0.3)$, i.e. the experimental proof that the nuclear dechanneling length does not depend on particle charge. Such result is fundamental for the design and fabrication of crystals suitable for the manipulation of both positive and negative particles. Moreover, the already existing experimental studies for negative particles may be extended to positive particles and vice versa. As an example, the nuclear de-channeling length for positrons at MAMI or SLAC may be inferred from the measured de-channeling length of electrons at the same energies, or the nuclear de-channeling length of anti-protons at $400 \mathrm{GeV} / \mathrm{c}$ from the measured de-channeling length of the protons.

Acknowledgements The authors acknowledge partial support by the Instituto Nazionale di Fisica Nucleare under the CHANEL and GECO projects and by the H2020 project AIDA-2020, GA no. 654168. The authors acknowledge the help and support of SPS-CERN coordinator and staff. We acknowledge the CINECA award under the ISCRA initia- tive, for the availability of high performance computing resources and support.

Open Access This article is distributed under the terms of the Creative Commons Attribution 4.0 International License (http://creativecomm ons.org/licenses/by/4.0/), which permits unrestricted use, distribution, and reproduction in any medium, provided you give appropriate credit to the original author(s) and the source, provide a link to the Creative Commons license, and indicate if changes were made.

Funded by SCOAP ${ }^{3}$.

\section{References}

1. V. Guidi, L. Lanzoni, A. Mazzolari, J. Appl. Phys. 107(11), 113534 (2010). doi:10.1063/1.3372722. http://link.aip.org/link/?JAP/107/ $113534 / 1$

2. V. Guidi, A. Antonini, S. Baricordi, F. Logallo, C. Malagu, E. Milan, A. Ronzoni, M. Stefancich, G. Martinelli, A. Vomiero, Nucl. Instrum. Meth. Phys. Res. Sect. B Beam Interact. Mater. Atoms. Relat. Channel. Relat. Coher. Phenom. Strong Fields 234(1), 40 (2005). doi:10.1016/j.nimb.2005.01.008. http://www. sciencedirect.com/science/article

3. A. Mazzolari, V. Guidi, D.D. Salvador, L. Bacci, Nucl. Instrum. Meth. Phys. Res. Sect. B Beam Interact. Mater. Atoms 309(0), 130 (2013). doi:10.1016/j.nimb.2013.03.002. http://www. sciencedirect.com/science/article

4. E. Bagli, L. Bandiera, V. Guidi, A. Mazzolari, D. Salvador, A. Berra, D. Lietti, M. Prest, E. Vallazza, Eur. Phys. J. C 74(1), 1 (2014). doi:10.1140/epjc/s10052-014-2740-7

5. A. Mazzolari, E. Bagli, L. Bandiera, V. Guidi, H. Backe, W. Lauth, V. Tikhomirov, A. Berra, D. Lietti, M. Prest, E. Vallazza, D. De Salvador, Phys. Rev. Lett. 112, 135503 (2014). doi:10.1103/ PhysRevLett.112.135503

6. U. Wienands, T.W. Markiewicz, J. Nelson, R.J. Noble, J.L. Turner, U.I. Uggerhøj, T.N. Wistisen, E. Bagli, L. Bandiera, G. Germogli, V. Guidi, A. Mazzolari, R. Holtzapple, M. Miller, Phys. Rev. Lett. 114, 074801 (2015). doi:10.1103/PhysRevLett.114.074801

7. V. Guidi, A. Mazzolari, D. De Salvador, L. Bacci, Phys. Rev. Lett. 108, 014801 (2012). doi:10.1103/PhysRevLett.108.014801

8. E. Bagli et al., Phys. Rev. Lett. 110, 175502 (2013). doi:10.1103/ PhysRevLett.110.175502

9. E. Bagli, V. Guidi, A. Mazzolari, L. Bandiera, G. Germogli, A.I. Sytov, D. De Salvador, A. Argiolas, M. Bazzan, A. Carnera, A. Berra, D. Bolognini, D. Lietti, M. Prest, E. Vallazza, Phys. Rev. Lett. 115, 015503 (2015). doi:10.1103/PhysRevLett.115.015503

10. T.N. Wistisen et al., Phys. Rev. Lett. 112, 254801 (2014). doi:10. 1103/PhysRevLett.112.254801

11. L. Bandiera, E. Bagli, G. Germogli, V. Guidi, A. Mazzolari, H. Backe, W. Lauth, A. Berra, D. Lietti, M. Prest, D. De Salvador, E. Vallazza, V. Tikhomirov, Phys. Rev. Lett. 115, 025504 (2015). doi:10.1103/PhysRevLett.115.025504

12. L. Bandiera, E. Bagli, V. Guidi, A. Mazzolari, A. Berra, D. Lietti, M. Prest, E. Vallazza, D. De Salvador, V. Tikhomirov, Phys. Rev. Lett. 111, 255502 (2013). doi:10.1103/PhysRevLett.111.255502

13. A.F. Elishev et al., Phys. Lett. B 88, 387 (1979). doi:10.1016/ 0370-2693(79)90492-1. http://www.sciencedirect.com/science/ article/

14. W. Scandale et al., Phys. Lett. B 692(2), 78 (2010). doi:10.1016/ j.physletb.2010.07.023. http://www.sciencedirect.com/science/ article/

15. A.G. Afonin et al., J. Exp. Theoret. Phys. Lett. 67(10), 781 (1998). doi:10.1134/1.567748 
16. A.S. Denisov et al., Nucl. Instrum. Methods Phys. Res. Sect. B 69,382 (1992). doi:10.1016/0168-583X(92)96034-V.http://www. sciencedirect.com/science/article/

17. W. Scandale et al., Lett Intent Exper LHC (CERN) 10, 2011 (2011)

18. W. Scandale, G. Arduini, M. Butcher, F. Cerutti, M. Garattini, S. Gilardoni, A. Lechner, R. Losito, A. Masi, D. Mirarchi, S. Montesano, S. Redaelli, R. Rossi, P. Schoofs, G. Smirnov, G. Valentino, D. Breton, L. Burmistrov, V. Chaumat, S. Dubos, J. Maalmi, V. Puill, A. Stocchi, E. Bagli, L. Bandiera, G. Germogli, V. Guidi, A. Mazzolari, S. Dabagov, F. Murtas, F. Addesa, G. Cavoto, F. Iacoangeli, L. Ludovici, R. Santacesaria, P. Valente, F. Galluccio, A. Afonin, Y. Chesnokov, A. Durum, V. Maisheev, Y. Sandomirskiy, A. Yanovich, A. Kovalenko, A. Taratin, A. Denisov, Y. Gavrikov, Y. Ivanov, L. Lapina, L. Malyarenko, V. Skorobogatov, T. James, G. Hall, M. Pesaresi, M. Raymond, Phys. Lett. B 758, 129 (2016). doi:10.1016/j.physletb.2016.05.004

19. J. Lindhard, Danske Vid. Selsk. Mat. Fys. Medd. 34, 14 (1965)

20. V.M. Biryukov, Y.A. Chesnekov, V.I. Kotov, Crystal Channeling and Its Applications at High-Energy Accelerators (Springer, Berlin, 1996)

21. W. Scandale et al., Phys. Lett. B 680(2), 129 (2009). doi:10.1016/ j.physletb.2009.08.046. http://www.sciencedirect.com/science/ article/

22. R.J. Carrigan, J. Ellison, Relativistic Channeling (Plenum Press, New York, 1987)

23. G. Arduini et al., Phys. Lett. B 422, 325 (1998). doi:10.1016/ S0370-2693(98)00132-4. http://www.sciencedirect.com/science/ article/

24. J.F. Bak et al., Nucl. Phys. B 242(1), 1 (1984). doi:10.1016/ 0550-3213(84)90130-5. http://www.sciencedirect.com/science/ article/

25. W.M. Gibson et al., Nucl. Instrum. Methods Phys. Res., Sect. B 2, 54 (1984). doi:10.1016/0168-583X(84)90154-X. http://www. sciencedirect.com/science/article/

26. W. Scandale et al., Phys. Lett. B 719, 70 (2013). doi:10.1016/ j.physletb.2012.12.061. http://www.sciencedirect.com/science/ article/

27. E. Tsyganov, Some aspects of the mechanism of a charge particle penetration through a monocrystal. Tech. rep., Fermilab (1976). (Preprint TM-682)

28. E. Tsyganov, Estimates of cooling and bending processes for charged particle penetration through a mono crystal. Tech. rep., Fermilab (1976). (Preprint TM-684)
29. A. Mazzolari et al., Proc. of 1st International Particle Accelerator Conference: IPAC'10 p. TUPEC080 (2010)

30. L. Celano et al., Nucl. Instrum. Methods Phys. Res., Sect. A 381(1), 49 (1996). doi:10.1016/0168-9002(96)00431-7. http:// www.sciencedirect.com/science/article/

31. S. Agostinelli et al., Nucl. Instr. Meth. Phys. Res. Sect. A Acceler. Spectr. Detect. Assoc. Equipm. 506(3), 250 (2003). doi:10.1016/ S0168-9002(03)01368-8. http://www.sciencedirect.com/science/ article/

32. J. Allison, K. Amako, J. Apostolakis, P. Arce, M. Asai, T. Aso, E. Bagli, A. Bagulya, S. Banerjee, G. Barrand, B. Beck, A. Bogdanov, D. Brandt, J. Brown, H. Burkhardt, P. Canal, D. Cano-Ott, S. Chauvie, K. Cho, G. Cirrone, G. Cooperman, M. Cortés-Giraldo, G. Cosmo, G. Cuttone, G. Depaola, L. Desorgher, X. Dong, A. Dotti, V. Elvira, G. Folger, Z. Francis, A. Galoyan, L. Garnier, M. Gayer, K. Genser, V. Grichine, S. Guatelli, P. Guèye, P. Gumplinger, A. Howard, I. Hřivnáčová, S. Hwang, S. Incerti, A. Ivanchenko, V. Ivanchenko, F. Jones, S. Jun, P. Kaitaniemi, N. Karakatsanis, M. Karamitrosi, M. Kelsey, A. Kimura, T. Koi, H. Kurashige, A. Lechner, S. Lee, F. Longo, M. Maire, D. Mancusi, A. Mantero, E. Mendoza, B. Morgan, K. Murakami, T. Nikitina, L. Pandola, P. Paprocki, J. Perl, I. Petrović, M. Pia, W. Pokorski, J. Quesada, M. Raine, M. Reis, A. Ribon, A.R. Fira, F. Romano, G. Russo, G. Santin, T. Sasaki, D. Sawkey, J. Shin, I. Strakovsky, A. Taborda, S. Tanaka, B. Tomé, T. Toshito, H. Tran, P. Truscott, L. Urban, V. Uzhinsky, J. Verbeke, M. Verderi, B. Wendt, H. Wenzel, D. Wright, D. Wright, T. Yamashita, J. Yarba, H. Yoshida, Nucl. Instr. Meth. Phys. Res. Sect. A Acceler. Spectr. Detect. Assoc. Equipm. 835, 186 (2016). doi:10.1016/j.nima.2016.06.125. http:// www.sciencedirect.com/science/article/

33. T.N. Wistisen, U.I. Uggerh øj, U. Wienands, T.W. Markiewicz, R.J. Noble, B.C. Benson, T. Smith, E. Bagli, L. Bandiera, G. Germogli, V. Guidi, A. Mazzolari, R. Holtzapple, S. Tucker, Phys. Rev. Accel. Beams 19, 071001 (2016). doi:10.1103/PhysRevAccelBeams.19. 071001

34. E. Bagli, M. Asai, D. Brandt, A. Dotti, V. Guidi, D.H. Wright, Eur. Phys. J. C 74(8), 2996 (2014). doi:10.1140/epjc/ s10052-014-2996-y

35. E. Bagli, V. Guidi, Nucl. Instrum. Meth. Phys. Res. Sect. B Beam Interact. Mater. Atoms 309(0), 124 (2013). doi:10.1016/j.nimb. 2013.01.073. http://www.sciencedirect.com/science/article/

36. E. Bagli, V. Guidi, V.A. Maisheev, Phys. Rev. E 81, 026708 (2010). doi:10.1103/PhysRevE.81.026708 